Open Scientist Handbook • OSH

\title{
The practical wisdom of doing science
}

Bruce R. Caron

Published on: Mar 10, 2021

DOI: $10.21428 / 8 b b b 7 f 85 . e c 1 e f 718$

License: Creative Commons Attribution 4.0 International License (CC-BY 4.0). 


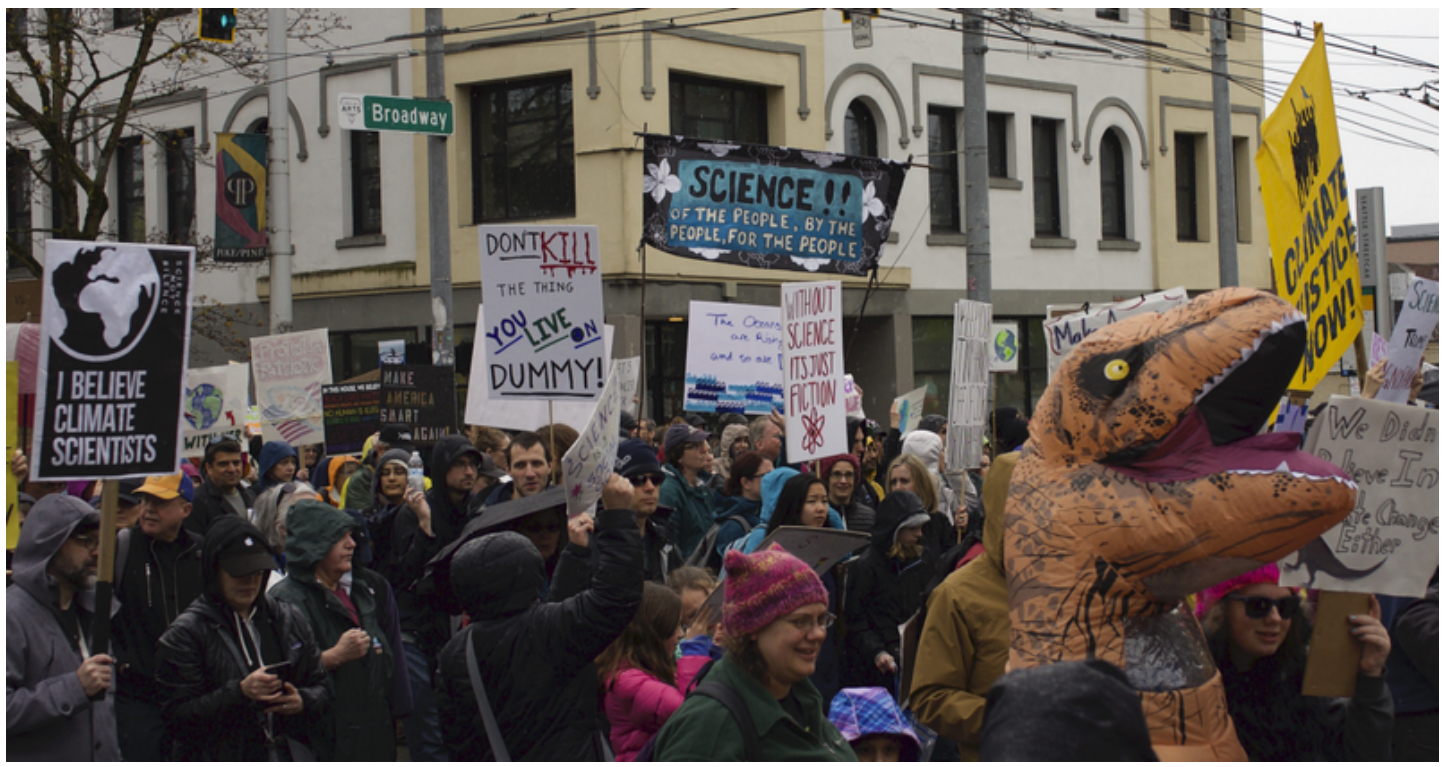

"If you compromise your integrity and principles on minor issues, it gets easier to make bad choices on the big issues" (Dunwoody and Collins 2015).

"Do the right thing. It will gratify some people and astonish the rest" (Mark Twain, attrib.).

The current research on "wisdom" defines this in approximately as many ways as there are wisdom researchers (Sternberg 1990). Fortunately, we do not need to lock down a definition of wisdom here to see its lack in the academy, and the benefits of promoting this as one more measure of what it means to do science and to be a scientist today. Most of the research on practical wisdom confirms what you already think about this. People who are wise in this way know how and when to do the right thing in a broad range of circumstances.

It's not all that simple, of course. Doing the right thing is not merely "being right," and most certainly is not thinking you're right, and convincing everyone else how wrong they are. Practical wisdom operates through the infinite play of open, complex intellectual and normative choices, and conflicting and ambiguous circumstances.

The "right thing" is rarely an easy binary operation and it often requires insights and emotional commitments beyond a simple logic. It may also be an action that goes against the immediate best interests of others or the wise person. "[M]any who have written on wisdom have identified it with the ability to develop and defend good 
judgments about the difficult, wicked-decision problems characteristic of adult life" (Kitchener and Brenner 1990).

Mostly, practical wisdom examples involve interpersonal or socioeconomic decisions. These examples map how individuals wend their life together with others and the world. This part of practical wisdom encompasses careers in and out of the academy. You can explore the more general forms of practical wisdom literature elsewhere (Practical wisdom: The right way to do the right thing by Schwartz and Sharpe (2010) is a good place to start, also Barry Schwartz's TED talk [Accessed January 7, 2020] or his WIRED interview). So, what forms of practical wisdom are peculiar to doing science?

In finite games, where arbitrary scarcity and external incentives warp the moral fabric of interaction, practical wisdom often gives way to self-promotion strategies. Somebody has to lose. Actually, most people need to lose in order for winning to matter. In the infinite play of science, nobody wins or loses, and the main strategies include sharing knowledge and adding new players to the mix. Science loads additional wicked-problem solving on top of the adult life problems of work and home. Wise researchers confront the silent unknowns of a complex and emergent natural world.

The ability to start from observations and data about the world and transform these into information, knowledge, and understanding is an inherently moral activity; each bit of new knowledge-big or small-changes existing rules for every scientist and expands the envelope of possible human action. Medical science practice is often cited as a key discipline in need of practical wisdom because of its everyday moral decision making (See: Branch and Mitchell 2011; Kaldjian 2010; and Jeste, et al. 2019). All science domains are similarly implicated when they enter into infinite play.

\section{How does practical wisdom improve research?}

Judith Glück (2017) makes a case for practical wisdom in the academy. The first behavior is a desire for a deep understanding of complex emergent systems, instead of a personal claim about some potentially universal truth. This is precisely the difference between the infinite play science, and playing a finite zero-sum game. "Over time, wise researchers' desire to thoroughly understand should lead them to develop an extraordinary amount of knowledge: a broad and deep integrative understanding of a subject matter that includes a keen awareness of what they do not (and may never) know" (ibid). In short, they become intellectually humble. 
“[Humble intelligence is] a method of thinking. It's about entertaining the possibility that you may be wrong and being open to learning from the experience of others. Intellectual humility is about being actively curious about your blind spots. One illustration is in the ideal of the scientific method, where a scientist actively works against her own hypothesis, attempting to rule out any other alternative explanations for a phenomenon before settling on a conclusion. It's about asking: What am I missing here?” (Resnick 2019; Accessed June 7, 2019).

Practical wisdom in the academy is built upon the humble intelligence of each scientist. As science becomes more collaborative, networking and teamwork bring new demands on the practical wisdom of every member. To review, Tangney (2000) proses that intellectual humility requires five abilities:

A. the ability to acknowledge mistakes and shortcomings;

B. openness to perspective and change;

C. an accurate view of the self's strengths;

D. ability to acknowledge and experience life outside the direct consciousness of the self; and,

E. the ability to appreciate the worth of all things.

Each of these abilities adds value to any academy collaboration effort. Open science captures this value by promoting equality of access and networked collaborative opportunities.

\section{Greater good: Delving into the Just Causes of science}

The second wise-researcher behavior is a high level of concern and care for the "greater good": for the welfare of the entire "Republic of Science" (Polanyi 1962), of the next generation of scientists, and of the planet:

"Wise researchers will be concerned with the well-being of others, ranging from their students to the world at large. Inside the university, wise researchers will care about the quality of their teaching. ... Wise researchers are also caring, generative mentors who seriously work on supporting the career development of their mentees and genuinely enjoy their success" (ibid).

The care that wise researchers bring to their science reflects their ability to set their own ego aside. Many of the distinctions between practical wisdom and general 
intellectual accomplishment pivot on this attention to the greater good: to the underlying reasons, the overarching effects, the larger, messier, more complex consequences of new knowledge.

Open-access science publishing can support the wise researcher looking to share their whole research in exchange for access to the research of their colleagues. New evaluation tools that reward ethical behaviors and a much greater variety of goals and social activities would support the efforts of those with a recognized concern for the welfare of their mentees and for the research success of others. Assessment tools that capture a diverse and incremental landscape of work outputs and socially-supportive activities are already being developed in the workplace outside the academy (See: Buckingham and Goodall 2019).

Practical wisdom is also an antidote to assholes in the academy. The Handbook deals with assholes elsewhere (See: The Zero-Asshole Zone). Most wisdom research concludes that you cannot have practical wisdom and also be an asshole or evil (See: Stanford Encyclopedia of Philosophy: Wisdom, 2002). In part, this is because you cannot have practical wisdom without actually using your practical wisdom. As noted elsewhere in the Handbook, much of the assholic behavior in the academy is learned and rewarded today; and so, most academy assholes are not irredeemable. They learn and use bad behavior to win the finite games currently infecting the academy. They can unlearn these behaviors and gain some practical wisdom over time. Some, however, are true assholes. These will resent and resist change when your new openscience cultural practices no longer support their bad behavior.

How do we build the practices of humble intelligence and a care for the greater good into the academy? Such is the project of this Handbook, a guide to changing culture in your precinct of the academy, wherever this is. Open science builds fierce equality into the academy as a normative behavior that expresses intellectual humility and inclusiveness. In the infinite play of science new knowledge can be found by anyone in the Republic of Science. Open science uses demand sharing to support the greater good. As a sharing economy, the academy's goods gain value across time and space.

The starting point for culture change is, as always, changing yourself. Everyone can gain practical wisdom, even tenured faculty. Start today. Be wiser tomorrow.

\section{Wisdom learning starts in childhood, but need not end there.}

Some researchers point out that a fair amount of practical wisdom is learned throughout childhood, mostly during play (See: Feist 2006; Brown 2009; Carlson and 
White 2013; and Sharma and Dewangan 2017). If you are no longer a child as you read this, do not worry, you can still catch up. To begin with, play is always available even for adults (See: < https://eachother.org.uk/the-right-to-play-adults/> Accessed January 5, 2020).

While the "skills" of practical wisdom-and wisdom in general-cannot be gained through the same type of specific practice as, say, a violin, or a golf swing, practical wisdom is similarly experience-based (Cantrell and Sharpe 2016). Like most cultural practices, you can get better at practical wisdom through practice, it is not an inherent trait. You are not born with all the wisdom you can have nor what you might really need to use for your career in the academy.

Glück and Bluck (2103) demonstrate a model for acquiring personal wisdom based on a "strong sense of mastery, high levels of openness, a reflective attitude, and emotion regulation skills combined with empathy." This "MORE" model can be used in "leadership" curriculum development at the undergraduate level (Sharma and Dewangan 2017). The Phronesis Project [Accessed January 8, 2020] at the University of Virginia School of Medicine is pioneering practical wisdom training in its curriculum. Just remember that you can also have fun while becoming wiser. You enjoy learning, after all, so learning practical wisdom is an opportunity to master another life skill:

"Another value of science is the fun called intellectual enjoyment which some people get from reading and learning and thinking about it, and which others get from working in it" (Feynman, et al. 2005).

Schwartz and Sharpe (2010) find that institutions that manage behaviors through rigid rules or laws crowd out opportunities for members to gain wisdom from self-governed interactions. "[I]t's important to resist those rules and incentives that eviscerate discretion and threaten wisdom. That's why we need to reform those institutions that are driving wisdom out." It could be that your institutions would benefit from more democracy and healthy arguments, and fewer rules. The Handbook sections on Learning Organizations are a good place to start.

\section{Bibliography: Open Scientist Handbook References}

